\title{
ОБОСНОВАНИЕ РЕПРЕЗЕНТАТИВНОГО ОБЪЕМА ДАННЫХ ФИЛЬТРАЦИОННО-ЕМКОСТНЫХ СВОЙСТВ ДЛЯ ПОЛУЧЕНИЯ СТАТИСТИЧЕСКИ ДОСТОВЕРНЫХ ПЕТРОФИЗИЧЕСКИХ СВЯЗЕЙ
}

\author{
А.А.АБРОСИМОВ, Е.В.ШЕЛЯГО, И.В.ЯЗЫНИНА
}

Российский государственный университет нефти и газа имени И.М.Губкина, Москва, Россия

\begin{abstract}
В статье рассмотрены вопросы обоснования объема выборки для петрофизического описания объекта по результатам традиционных лабораторных измерений, а также обработки данных рентгеновской томографии. Рассмотрен новый подход к расчету фильтрационно-емкостных свойств коллекторов из данных метода рентгеновской томографии путем формирования массива виртуальных кубов. Обсуждаются вопросы необходимого количества выделяемых кубов для моделирования флюидодинамики. Показаны критерии числа лабораторных измерений и выделяемых из цифровой модели виртуальных кубов для получения статистически достоверных петрофизических связей. Сделан вывод о необходимости корректного сравнения расчетных и лабораторных петрофизических связей.

Ключевые слова: анализ керна; рентгеновская томография; петрофизические связи; выборка; пористость; проницаемость; виртуальный куб
\end{abstract}

Как цитировать эту статью: Абросимов А.А. Обоснование репрезентативного объема данных фильтрационно-емкостных свойств для получения статистически достоверных петрофизических связей / А.А.Абросимов, Е.В.Шеляго, И.В.Язынина // Записки Горного института. 2018. Т. 233. С. 487-491. DOI: 10.31897/PMI.2018.5.487

Введение. Первые работы в области использования рентгеновской томографии (РТ) для изучения горных пород, в том числе коллекторов нефти и газа, относятся к 80-м годам прошлого века [15]. В настоящее время данный метод вызывает повышенный интерес у нефтегазовых компаний, который связан, с одной стороны, с тем, что он является неразрушающим, требует для исследования малый объем образца любой формы и, следовательно, открывает перспективы исследования слабо консолидированного и непригодного для лабораторных исследований керна. С другой стороны, математическое моделирование позволяет рассчитывать необходимые для петрофизиков и разработчиков свойства. Также интересна идея создания «виртуального кернохранилища» с целью его использования для подбора методов повышения нефтеотдачи.

Количество работ, использующих РТ для исследований керна продуктивных нефтегазоносных отложений, растет. Сегодня, как правило, томография дополняет литологическое описание керна и применяется для количественной оценки пористости $[3,5,10]$. Расчет фильтрационных характеристик (например, газопроницаемости) встречается реже [7, 11], а расчетные капиллярные кривые или кривые относительных фазовых проницаемостей - в единичных случаях [9]. Исследователи сталкиваются с ограничениями математических алгоритмов, большими временными затратами на проведение расчетов, а также высокими требованиями к вычислительным мощностям.

Для расчета фильтрационно-емкостных свойств (ФЕС) существуют несколько подходов (рис.1). Первый заключается в подборе представительного объема (в англ. REV - representative elementary volume) и использовании его в гидродинамическом моделировании в масштабе пор [1, $6,8,12-14]$. Использование данного подхода требует значительно больших вычислительных ресурсов, в связи с чем был предложен альтернативный подход с формированием значительно меньших объемов (виртуальных кубов) [2, 4]. Уменьшение виртуального куба снижает требования к вычислительной машине, но и уменьшает его представительность по отношению к исходному образцу. Поэтому авторами данной статьи предложено выделять массивы виртуальных кубов из одного образца керна. Данный подход позволяет экономить вычислительные ресурсы и время работы рентгеновской трубки для получения конечного результата, а также построить петрофизические связь по ограниченному числу стандартных цилиндров керна или по фрагментам в случае его разрушенности.

В настоящей работе приведено обоснование выбора необходимого количества виртуальных кубов для построения статистически достоверной петрофизической связи по данным РТ. 


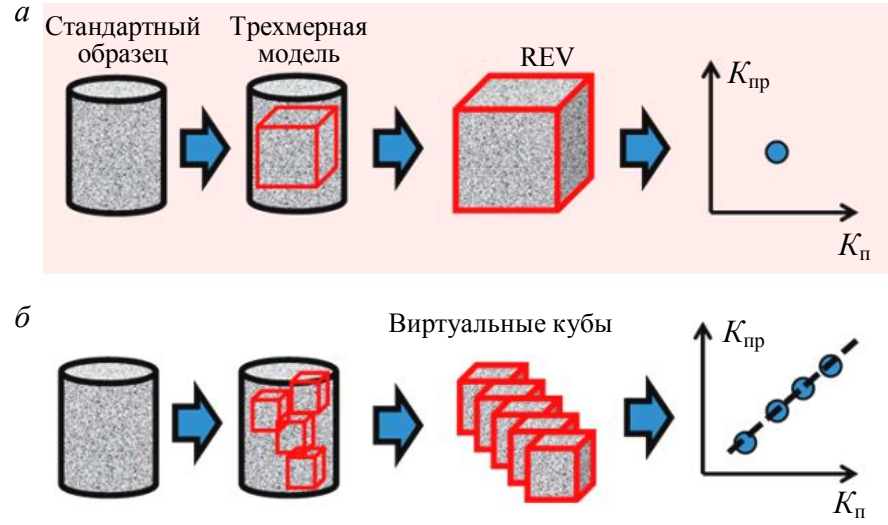

Рис.1. Подходы к расчету ФЕС горных пород $a$ - традиционный подход - одно значение (суперкомпьютер); $\sigma$ - новый подход - несколько значений (стационарный компьютер)

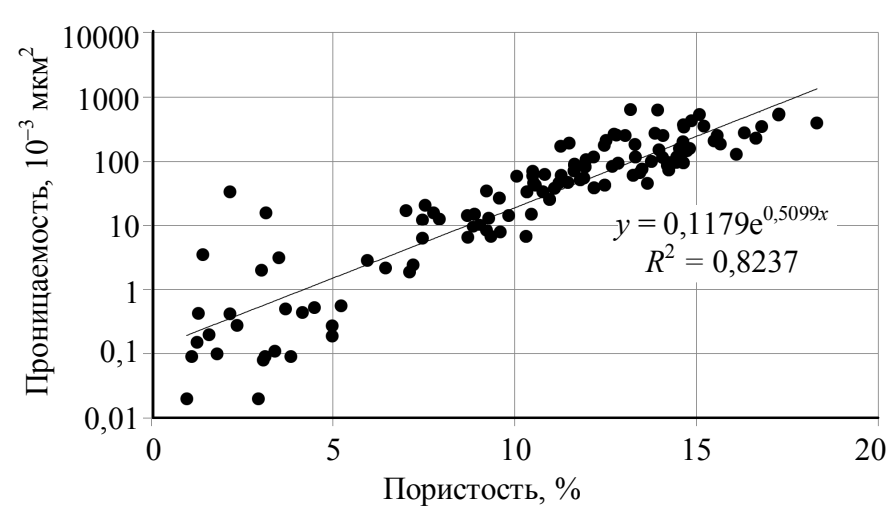

Рис.2. Зависимость $K_{\text {пр }}=f\left(K_{\text {п) }}\right)$ для отложения среднего девона афонинского горизонта

Объект и метод исследования. Объектом исследования являются терригенные отложения среднего девона афонинского горизонта. Лабораторные исследования проведены по 150 образцам, для РТ исследований были отобраны два образца, представленные песчаником мелко- и среднезернистым, по минеральному составу кварцевым (90-95 \%). Пустотное пространство представлено порами межзернового типа и распределено равномерно.

Метод рентгеновской томографии заключается в использовании проникающей способности однонаправленного рентгеновского излучения с последующей регистрацией и построением рентгеновского изображения на приемниках рентгеновских лучей. Результатом РТ-съемки и последующей обработки является трехмерная модель керна. Для оценки фильтрационноемкостных свойств выделяется пустотное пространство и строится его объемная модель. В рамках предложенного подхода предлагается разбивать модель пустотного пространства отснятого образца на фрагменты (виртуальные кубы), которые используются для расчета пористости и газопроницаемости и построения соответствующей петрофизической зависимости $K_{\text {пр }}=f\left(K_{\text {пा }}\right)$.

Число виртуальных кубов изменяется до тех пор, пока полученная по ним петрофизическая зависимость не перестанет изменяться и наилучшим образом не совпадет с зависимостью, полученной в лаборатории на образцах 30×30 мм в соответствии с ГОСТ 26450.2-85 «Породы горные. Метод определения коэффициента абсолютной газопроницаемости при стационарной и нестационарной фильтрации». В качестве параметров, контролирующих изменение зависимости, были выбраны: дисперсия, среднее квадратичное отклонение (СКО) и тангенс угла наклона петрофизической связи.

Результаты исследования, обсуждение. Согласно РД 153-39.0-072-01 «Техническая инструкция по проведению геофизических исследований и работ приборами на кабеле в нефтяных и газовых скважинах» для построения петрофизических связей «керн-керн» необходимо исследовать не менее 30 образцов керна. В связи с этим было решено вначале проверить это положение на лабораторной зависимости (рис.2), где выборка образцов производилась случайным образом.

На рис.3 показано, как происходит изменение значений дисперсии, среднего квадратичного отклонения (CKO) и тангенса угла в зависимости от размера выборки. Для согласования значений проницаемости удается добиться стабилизации параметров при 40 образцах керна, в то время как для пористости для этого необходимо 80 образцов. Тангенс угла наклона является комплексным параметром, учитывающим взаимоотношение обоих параметров, и, как видно, перестает изменяться при количестве исследуемых образцов, равным 80. Таким образом, для данного типа коллектора количество образцов, необходимых для построения статистически достоверной связи проницаемости от пористости, составляет порядка 80, что согласуется с правилами РД (более 30 образцов). Если взять для исследований только 30-40 образцов, то можно получить некорректные данные, поэтому рекомендуется проводить подобный статистический анализ, который, в свою очередь, может являться дополнением к указанному РД. 


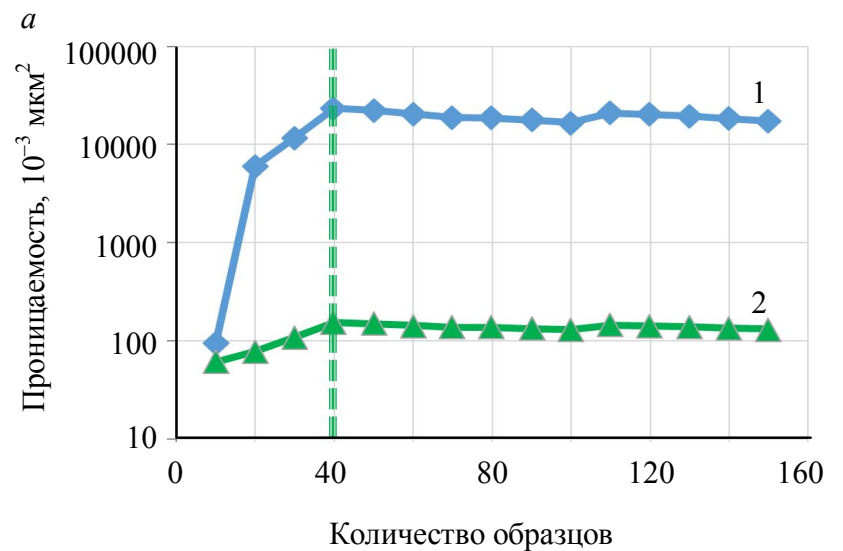

$\sigma$

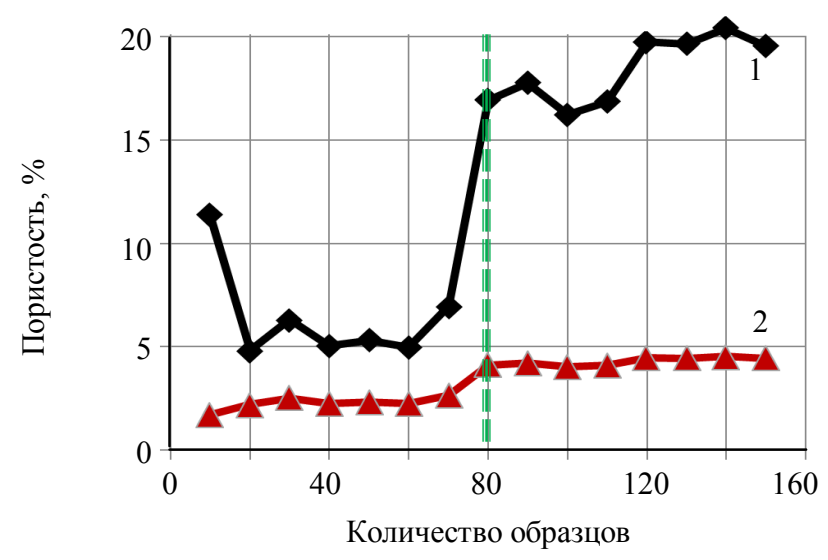

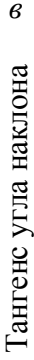

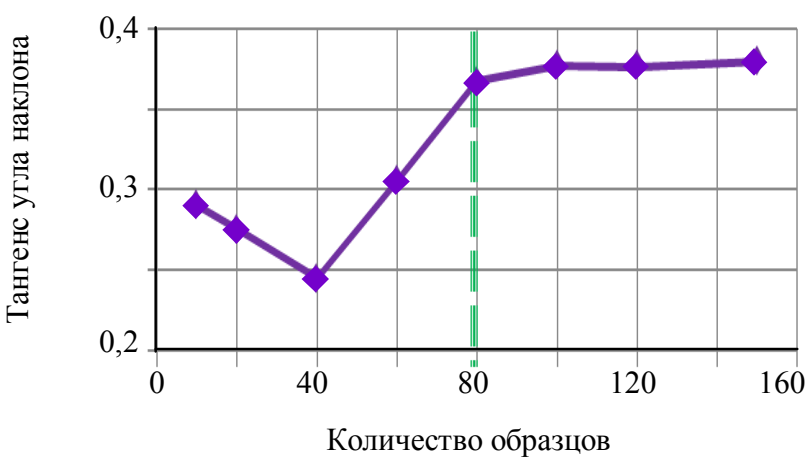

Рис.3. Зависимости дисперсии и среднего квадратичного отклонения проницаемости $(a)$, пористости (б), тангенса угла (в) от количества исследованных образцов

$$
1 \text { - дисперсия; } 2 \text { - СКО }
$$

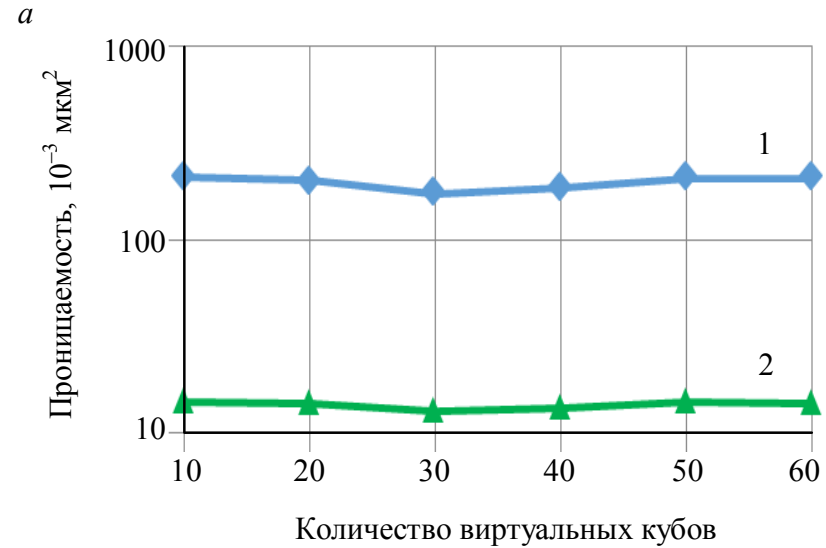

6
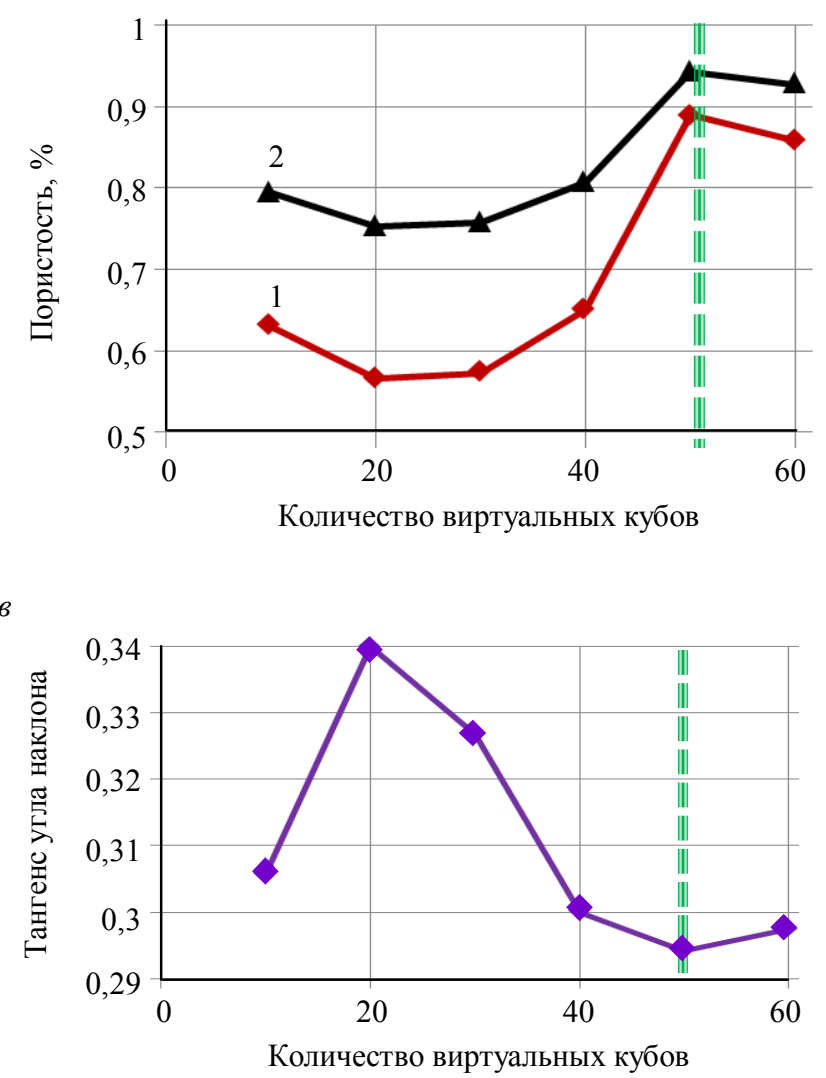

Рис.4. Зависимости дисперсии и среднего квадратичного отклонения проницаемости (a), пористости (б), тангенса угла (в) от количества виртуальных кубов, 1 - дисперсия; 2 - СКО

Аналогичные процедуры были проведены в случае РТ, только в данном случае зависимость строилась внутри одного образца. Предположение о том, что виртуальные кубы, вырезанные из данных РТ-съемки одного образца керна, могут формировать корреляционную связь пористости и проницаемости, является новым и не применялось до сих пор на практике. На рис. 4 приводятся результаты по одному из исследованных образцов.

Как видно, в случае проницаемости дисперсия и СКО практически не меняются с увеличением количества виртуальных кубов. Для пористости, также как и для тангенса угла наклона, эти значения устанавливаются на постоянном уровне при 50 кубах. Однако для второго исследуемого образца это количество составило 40 кубов, что говорит о том, что количество выделяемых кубов из каждого образца для построения статистически достоверной связи индивидуально. В результате применения данного подхода были получена расчетная связь пористость - прони- 


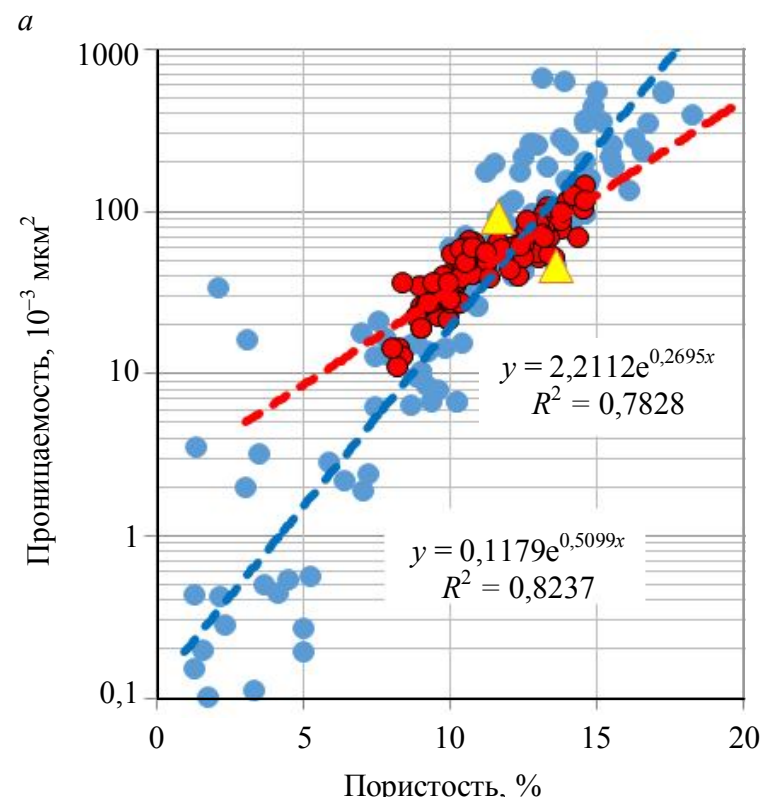

$\sigma$

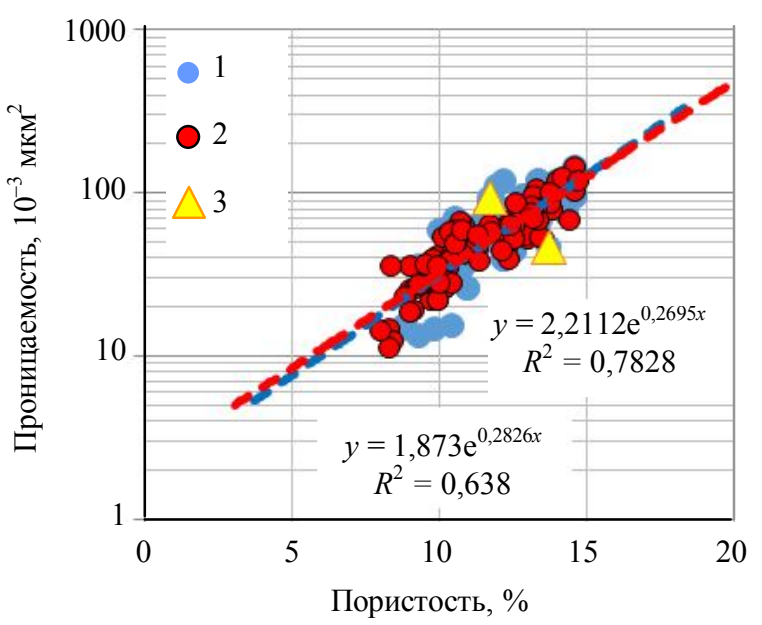

Рис.5. Сопоставление результатов: $a-$ с полным объемом лабораторных исследований $\sigma$ - в интервале исследований, полученных по РТ 1 - лаборатория; 2 - РТ; 3 - образцы для РТ

цаемость, сравнение ее с лабораторной приводится на рис.5, $a$. В целом, можно отметить хорошее совпадение результатов, однако зависимость, полученная по РТ, имеет несколько другой наклон. Это можно объяснить тем, что выбранные образцы (желтые треугольники) характеризуют достаточно узкую область лабораторной зависимости, поэтому сравнивать результаты по этим двум образцам с лабораторной зависимостью, имеющей значительно более широкий разброс, не совсем корректно. В связи с этим интервал сравнения был ограничен областью, где имелись расчетные данные РТ. На рис.5, б показано, что при этом достигается полная сходимость петрофизических связей.

\section{Выводы}

В рамках настоящей работы сформулированы основные составляющие статистического подхода к определению фильтрационно-емкостных свойств горных пород по данным РТ с использованием массива виртуальных кубов:

1. Приведено обоснование количества вырезаемых кубов на основе использования таких статистических параметров как дисперсия, среднее квадратичное отклонение и тангенс угла наклона. Данный подход является универсальным, поскольку может быть применим как для терригенных, так и для карбонатных образцов.

2. Показано, что предлагаемый подход также применим для лабораторных исследований керна, помогает более обоснованно подходить к выбору количества образцов для построения статистически достоверных связей и является дополнением к РД 153-39.0-072-01.

3. Надежное построение петрофизических связей с использованием статистического подхода по данным виртуальных кубов, созданных на базе РТ съемки, возможно только в пределах получаемых значений ФЕС. Аппроксимация расчетных зависимостей для фрагментов в область лучших или ухудшенных ФЕС может привести к погрешностям.

\section{ЛИТЕРАТУРА}

1. Абросимов А.А. Применение рентгенотомографии для изучения фильтрационно-емкостных систем коллекторов нефти и газа // Труды РГУ нефти и газа имени И.М.Губкина. 2015. № 4/281. С. 5-15.

2. Апробация нового подхода к определению петрофизических связей по данным рентгеновской томографии / И.В.Язынина, Е.В.Шеляго, А.А.Абросимов, Н.А.Веремко, Е.А.Грачев, Д.А.Бикулов // Нефтяное хозяйство. 2017. № 2. С. 3640. DOI: $10.24887 / 0028-2448-2017-2-36-40$.

3. Кочнев А.А. Палеонтологические исследования с помощью метода рентгеновской томографии / А.А.Кочнев, С.Н.Кривощеков // Master's Journal. 2015. № 2. С. 177-181.

4. Новый подход к исследованиям керна с помощью рентгеновской томографии для решения задач петрофизики / И.В.Язынина, Е.В.Шеляго, А.А.Абросимов, Н.А.Веремко, Н.Е.Грачев, Д.С.Сенин // Нефтяное хозяйство. 2017. № 1. C. $19-23$.

5. Чугунов С.С. Комплексирование методов рентгеновской томографии и трехмерной электронной микроскопии при исследовании пород баженовской свиты Западной Сибири / С.С.Чугунов, А.В.Казак, А.Н.Черемисин // Нефтяное хозяйство. 2015. №10. C. 44-49. 
Обоснование репрезентативного объема данных...

6. Al-Raoush R. Representative elementary volume analysis of porous media using X-ray computed tomography / R.Al-Raoush, A.Papadopoulos // Powder Technology. 2010. Vol. 200. P. 69-77.

7. Alreshedan F. Investigation of permeability, formation factor, and porosity relationships for Mesaverde tight gas sandstones using random network models / F.Alreshedan, A.J.Kantzas // Petrol Explor Prod Technol. 2016. Vol. 6. P. 545-554. DOI.org/10.1007/s13202-015-0202-x.

8. Effects of sampling volume on the measurement of soil physical properties: simulation with X-ray tomography data / P.Baveye, H.Rogasik, O.Wendroth, I.Onasch, J.W.Crawford // Measurement Science and Technology. 2002. Vol. 13. P. $775-784$.

9. Gas-Water Two Phase Flow Simulation Based on Pore Network Model / M.Mutailipu, Y.Liu, B.Wu, Y.Song, D.Wang, L.Ai. // Energy Procedia. 2017. Vol. 142. P. 3214-3219.

10. Hickman-Lewis K. X-ray microtomography as a tool for investigating the petrological context of Precambrian cellular remains // Geological Society. London. Special Publications. 2016. Vol. 448 (1). P. 33-56.

11. Permeability estimation of porous media by using an improved capillary bundle model based on micro-CT derived pore geometries / L.Jiang, Y.Liul, Y.Teng, J.Zhao1, Y.Zhang, M.Yang, Y.Song // Heat Mass Transfer. 2017. Vol. 53. P. 49-58. DOI 10.1007/s00231-016-1795-4.

12. Razavi M.R.B. Representative elementary volume analysis using X-ray computed tomography/ M.R.B.Razavi, B.Muhunthan, O.Al Hattamleh // Geotechnical Testing Journal. 2007. Vol. 30 (3). P. 212-219.

13. Various estimates of Representative Volume Element sizes based on a statistical analysis of the apparent behaviour of random linear composites / M.Salmi, F.Auslender, M.Bornert, M.Fogli // Comptes Rendus Mécanique. 2012. Vol. 340 (4-5). P. 230-246.

14. Vandenbygaart A.J. The representative elementary area (REA) in studies of quantitative soil micromorphology / A.J.Vandenbygaart, R.Protz // Geoderma. 1999. Vol. 89 (3-4). P. 333-346.

15. Wellington S.L. X-ray computerized tomography / S.L.Wellington, H.J.Vinegar // Journal of Petroleum Technology. 1987. Vol. 39. P. 885-898.

Aвторы: Абросимов А.А., канд. техн. наук, инженер, abrosimov.aа@inbox.rи (Российский государственный университет нефти и газа имени И.М.Губкина, Москва, Россия), Шеляго Е.В., канд. техн. наук, доцент, thelgp@yandex.ru (Российский государственный университет нефти и газа имени И.М.Губкина, Москва, Россия), Язынина И.В., канд. геол.минерал. наук, доиент, уаzупіпа@таіl.ru (Российский государственный университет нефти и газа имени И.М.Губкина, Москва, Россия).

Статья поступила в редакииюю 27.03.2018.

Статья принята к публикации 28.04.2018. 